\title{
Spintronic single-qubit gate based on a quantum ring with spin-orbit interaction
}

\author{
Péter Földi, ${ }^{1,2}$ Balázs Molnár, ${ }^{2}$ Mihály G. Benedict, ${ }^{1,2}$ and F. M. Peeters ${ }^{2}$ \\ ${ }^{1}$ Department of Theoretical Physics, University of Szeged, Tisza Lajos körút 84, H-6720 Szeged, Hungary \\ ${ }^{2}$ Departement Fysica, Universiteit Antwerpen (Campus Drie Eiken), Universiteitsplein 1, B-2610 Antwerpen, Belgium
}

(Received 11 June 2004; published 12 January 2005)

\begin{abstract}
In a quantum ring connected with two external leads, the spin properties of an incoming electron are modified by the spin-orbit interaction, resulting in a transformation of the qubit state carried by the spin. The ring acts as a one-qubit spintronic quantum gate whose properties can be varied by tuning the Rashba parameter of the spin-orbit interaction, by changing the relative position of the junctions, as well as by the size of the ring. We show that a large class of unitary transformations can be attained with already one ring-or a few rings in series-including the important cases of the $Z, X$, and Hadamard gates. By choosing appropriate parameters the spin transformations can be made unitary, which corresponds to lossless gates.
\end{abstract}

DOI: 10.1103/PhysRevB.71.033309

The electron spin degree of freedom is one of the prospective carriers ${ }^{1,2}$ of qubits, the fundamental units in quantum information processing. In order to implement quantum operations on electron spins, appropriate gates are necessary that operate on this type of qubits. We note that in the present context the word "gate" stands for an elementary logical operation. ${ }^{3}$ In this paper we show that a one-dimensional ring $^{4}$ connected with two external leads made of a semiconductor structure, ${ }^{5}$ such as InGaAs in which Rashba-type ${ }^{6}$ spin-orbit interaction is the dominant spin-flipping mechanism, can render such a gate. Conductance properties of this kind of ring have been discussed earlier in the case of diametrically connected leads. ${ }^{7-10}$

By taking here a different point of view, we focus explicitly on the spin transformation characteristics of this device, and show that those can be appropriately controlled by varying its geometrical and physical parameters in the experimentally feasible range. ${ }^{7,8}$ We shall determine the effects of changing the radius and the relative positions of the junctions, as well as the influence of varying the strength of the spin-orbit interaction via an external electric field. The conditions under which the incoming and transmitted spinors are connected unitarily will be determined, leading in principle to a lossless single-qubit gate. By connecting a few such rings in an appropriate manner, one can achieve practically all the important one-qubit gates. ${ }^{3}$

We consider a ring of radius $a$ in the $x-y$ plane and assume a tunable static electric field ${ }^{7}$ in the $z$ direction characterized by the parameter $\alpha$. Then, the spin-dependent Hamiltonian ${ }^{9,11}$ of a charged particle of effective mass $m^{*}$ is $H=\hbar \Omega\left[\left(-i \frac{\partial}{\partial \varphi}+\frac{\omega}{2 \Omega}\left(\sigma_{x} \cos \varphi+\sigma_{y} \sin \varphi\right)\right)^{2}-\frac{\omega^{2}}{4 \Omega^{2}}\right]$

where $\varphi$ is the azimuthal angle of a point on the ring, $\hbar \Omega=\hbar^{2} / 2 m * a^{2}$ is the parameter characterizing the kinetic energy of the charge, and $\omega=\alpha / \hbar a$ is the frequency associated with the spin-orbit interaction. Apart from constants, the Hamiltonian (1) is the square of the sum of the $z$ component of the orbital angular momentum operator $L_{z}=-i(\partial / \partial \varphi)$, and of $(\omega / \Omega) S_{r}$, where $S_{r}=\sigma_{r} / 2$ is the radial component of the spin (both measured in units of $\hbar$ ). $H$ commutes in a nontrivial way with $K=L_{z}+S_{z}$, the $z$ compo-
PACS number(s): 85.35.Ds, 03.67.-a, 71.70.Ej

nent of the total angular momentum. $H$ also commutes with $S_{\theta \varphi}=S_{x} \sin \theta \cos \varphi+S_{y} \sin \theta \sin \varphi+S_{z} \cos \theta$, the spin component in the direction determined by the angles $\theta$, and $\varphi$, where $\theta$ is given by

$$
\tan \theta=-\omega / \Omega \text {. }
$$

One easily can prove that the commutator $\left[K, S_{\theta \varphi}\right]=0$; therefore, we may look for simultaneous eigenstates of $H, K$ and $S_{\theta \varphi}$. In the $|+\rangle,|-\rangle$ eigenbasis of $S_{z}$ one finds these in the form

obeying

$$
\psi(\kappa, \varphi)=e^{i \kappa \varphi}\left(\begin{array}{c}
e^{-i \varphi / 2} u(\kappa) \\
e^{i \varphi / 2} v(\kappa)
\end{array}\right)
$$

$$
K \psi(\kappa, \varphi)=\kappa \psi(\kappa, \varphi),
$$

$$
S_{\theta \varphi} \psi(\kappa, \varphi)=s(\kappa) \psi(\kappa, \varphi), \quad s(\kappa)= \pm 1 / 2,
$$

and the energy eigenvalues are

$$
E=\hbar \Omega\left[\kappa^{2}-\mu \kappa w+1 / 4\right], \quad \mu= \pm 1,
$$

with $w=\sqrt{1+\left(\omega^{2} / \Omega^{2}\right)}$. In a closed ring $\kappa \pm 1 / 2$ must be an integer, while if one considers leads connected to the ring, there is no such restriction: the energy is a continuous variable, and then the possible values of $\kappa$ are the solutions of Eq. (5), which can be written as

$$
\kappa_{j}^{\mu}=\mu\left(w / 2+(-1)^{j} q\right), \quad j=1,2, \quad \mu= \pm 1,
$$

where $q=\sqrt{(\omega / 2 \Omega)^{2}+E / \hbar \Omega}$. The energy eigenvalues are fourfold degenerate: $j=1,2$ correspond to two distinct values of $\left|\kappa_{j}^{\mu}\right|$, while the additional degeneracy at a given $j$ is resolved by the sign of $\kappa_{j}^{\mu}$. The components of the eigenvectors in (3) are related as

$$
\frac{v\left(\kappa_{j}^{\mu}\right)}{u\left(\kappa_{j}^{\mu}\right)}=(\tan \theta / 2)_{\mu}=\frac{\Omega}{\omega}(1-\mu w) ;
$$

thus, this ratio depends only on the index $\mu$ of $\kappa_{j}^{\mu}$. The two possible eigenvalues in Eq. (4b) are $s\left(\kappa_{j}^{\mu}\right)=\mu / 2$; accordingly, the eigenstates for a given energy can be classified by giving the absolute value of $\kappa_{j}^{ \pm}$, together with the sign of the eigenvalue of $S_{\theta \varphi}$.

The stationary states of the problem, ring plus leads, 


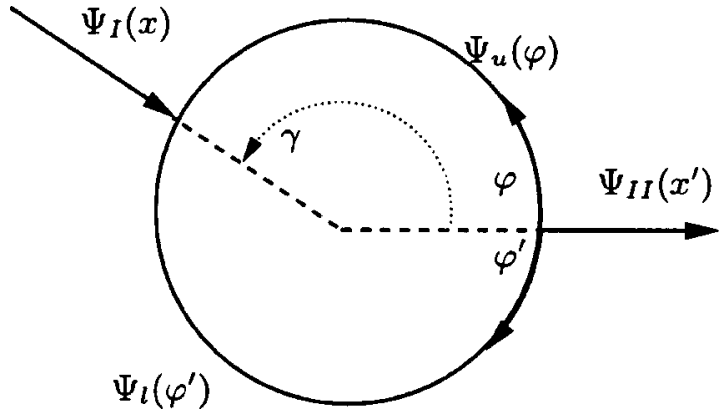

FIG. 1. The geometry of the device and the relevant wave functions in the different domains.

can be determined by fitting the solutions obtained in the different domains. Using local coordinates as shown in Fig. 1, the incoming wave, $\Psi_{I}(x)$, and the outgoing wave $\Psi_{I I}\left(x^{\prime}\right)$, are built up as linear combinations of spinors with spatial dependence $e^{i k x}$ etc., corresponding to $E=\hbar^{2} k^{2} / 2 m^{*}$

$$
\Psi_{I}(x)=\left(\begin{array}{l}
f_{1} \\
f_{2}
\end{array}\right) e^{i k x}+\left(\begin{array}{c}
r_{1} \\
r_{2}
\end{array}\right) e^{-i k x}, \quad \Psi_{I I}\left(x^{\prime}\right)=\left(\begin{array}{c}
t_{1} \\
t_{2}
\end{array}\right) e^{i k x^{\prime}} .
$$

The wave functions belonging to the same energy in the upper and lower arms of the ring can be written as linear combinations of the corresponding four eigenspinors

$$
\Psi_{u}(\varphi)=\sum_{\substack{j=1,2 \\ \mu= \pm}} a_{j}^{\mu} \psi\left(\kappa_{j}^{\mu}, \varphi\right) ; \quad \Psi_{l}\left(\varphi^{\prime}\right)=\sum_{\substack{j=1,2 \\ \mu= \pm}} b_{j}^{\mu} \psi\left(\kappa_{j}^{\mu},-\varphi^{\prime}\right)
$$

respectively. According to Fig. 1 the incoming wave at $x$ $=0$ is fitted to $\Psi_{u}$ at $\varphi=\gamma$ and to $\Psi_{l}$ at $\varphi^{\prime}=2 \pi-\gamma$, while the outgoing wave at $x^{\prime}=0$ is fitted to $\Psi_{u}$ and $\Psi_{l}$ at $\varphi=\varphi^{\prime}=0$. One has to require the continuity of the wave functions, as well as a vanishing spin current density at the two junctions. ${ }^{9,12,13}$ The resulting set of linear equations leads to a relation between the expansion coefficients in the different domains. The detailed procedure for the case $\gamma=\pi$ was described in Ref. 9 using the eigenbasis of $S_{\theta \varphi}$ at both junctions. As we will show, the more general geometry shown in Fig. 1 allows a significantly wider class of spin transformations to be described now in the fixed $S_{z}$ basis, which is more suitable to discuss the qubit operations. We focus here on the transmission properties of the ring, and obtain in the $|+\rangle,|-\rangle$ basis

with

$$
\begin{gathered}
\left(\begin{array}{l}
t_{1} \\
t_{2}
\end{array}\right)=T\left(\begin{array}{l}
f_{1} \\
f_{2}
\end{array}\right)=\left(\begin{array}{ll}
T_{11} & T_{12} \\
T_{21} & T_{22}
\end{array}\right)\left(\begin{array}{l}
f_{1} \\
f_{2}
\end{array}\right), \\
T=\left|T_{\gamma}\right| e^{i \delta_{0} / 2} e^{-i \gamma / 2} U,
\end{gathered}
$$

where the matrix elements of $U$ are $u_{11}=u_{2_{2}^{2}}^{*}$ $=\left[e^{-i \delta / 2} \sin ^{2}(\theta / 2)+e^{i \delta / 2} \cos ^{2}(\theta / 2)\right] e^{i \gamma / 2}, \quad u_{12}=-u_{21}^{*}$ $=i \sin (\delta / 2) \sin \theta e^{-i \gamma / 2} .\left|T_{\gamma}\right|$ and the phases $\delta_{0}$ and $\delta$ are obtained from

$$
\begin{gathered}
\left|T_{\gamma}\right| e^{i \delta_{ \pm}}=\frac{4 i k a q\left[\sin q(2 \pi-\gamma)+e^{i \Phi_{ \pm}} \sin q \gamma\right] e^{-i \gamma \Phi_{ \pm} / 2 \pi}}{k^{2} a^{2}\{\cos 2 q(\pi-\gamma)-\cos 2 q \pi\}+4 q^{2}\{\cos \Phi-\cos 2 q \pi\}+4 i k a q \sin 2 q \pi}, \\
\delta_{0}=\delta_{+}+\delta_{-}, \quad \delta=\delta_{+}-\delta_{-}=2 \arctan \frac{\sin (w \gamma / 2) \sin q(2 \pi-\gamma)+\sin w(\pi-\gamma / 2) \sin q \gamma}{\cos w(\pi-\gamma / 2) \sin q \gamma-\cos (w \gamma / 2) \sin q(2 \pi-\gamma)},
\end{gathered}
$$

where $\cos \Phi_{+}=\cos \Phi_{-} \equiv \cos \Phi$, with $\Phi_{ \pm}=\pi(-1 \pm w)$, the Aharonov-Casher phases ${ }^{14}$ for the corresponding spin directions.

The important fact is that $U$ is a unitary, unimodular matrix. It is this unitary part that performs a nontrivial spin transformation in the qubit space. In Eq. (11) $\left|T_{\gamma}\right|$ is a non-negative constant with $\left|T_{\gamma}\right| \leqslant 1$, which can be considered as the efficiency of the gate. Therefore, one has in general $\left|t_{1}\right|^{2}+\left|t_{2}\right|^{2} \leqslant 1$; nevertheless, the transmitted amplitudes can be renormalized, and their absolute value squared give the probabilities of having the corresponding spin direction, if the particle is assumed to be transmitted at all. In certain cases, to be discussed below, we find, however, that $T$ is unitary: $\left|T_{\gamma}\right|=1$. We shall turn now to analyze the transformation properties of this device in more detail.

If the incoming and outgoing leads are connected to the ring diametrically, then $\gamma=\pi$, and as seen from (13) also $\delta$ $=\pi$ independently from the energy. The transmission matrix then takes the form

$$
T=\left|T_{\pi}\right| e^{i\left(\delta_{0}+\pi\right) / 2}\left(\begin{array}{cc}
\cos \theta & -\sin \theta \\
\sin \theta & \cos \theta
\end{array}\right)=\left|T_{\pi}\right| e^{i\left(\delta_{0}+\pi\right) / 2} U,
$$

with

$$
\left|T_{\pi}\right| e^{i \delta_{0} / 2}=\frac{8 i k a q \sin (\pi q) \cos (\Phi / 2)}{k^{2} a^{2}(1-\cos 2 q \pi)+4 q^{2}(\cos \Phi-\cos 2 q \pi)+4 i k a q \sin 2 q \pi} .
$$


The unitary part $U$ of the transformation given by Eq. (14) is independent of the wave vector $k$, and it rotates the spin around the $y$ axis $^{3}$ by an angle $2 \theta$. By changing the strength of the spin-orbit interaction ${ }^{7} \omega=\alpha / \hbar a$, according to Eq. (2) the values of $|\theta|$ can be varied from 0 up to $0.8(\pi / 2)$. Figure 2 shows the gate efficiency $\left|T_{\pi}\right|$ as a function of $|\theta|$ and of $k a$ around $k_{F} a=20.4$, corresponding to a ring of radius $0.25 \mu \mathrm{m}$ and a Fermi energy $11.13 \mathrm{meV}$ of InGaAs. One sees also that for several values of $k a$ and $\theta$ the transformation is strictly unitary, with $\left|T_{\pi}\right|=1$. If one couples such unitary devices in series, then obviously the resulting transformation will be the product of the corresponding unitary matrices, and will be unitary again.

In the language of quantum informatics, ${ }^{3}$ the transformation (14) above represents a rather general single-qubit gate, and it shows that in principle a continuous set of spin rotations can be achieved with already a single diametrically connected ring. This can be further extended by coupling two or more such rings in series. A transformation of the form (14) with $\theta=\pi / 4$ is essentially a so-called Hadamard gate, ${ }^{3}$ which plays a distinguished role in quantum algorithms, while two such gates in series results in an $X$ gate or quantum NOT gate. Strictly speaking, in both cases one has to introduce an additional relative phase between the components, in order to have the correct determinant $(-1)$ of the transformations. ${ }^{3}$ This is possible with $\gamma \neq \pi$, to be discussed below.

In the case of arbitrary $\gamma$, other types of transformations can be realized. An important particular case is when $\delta=0$, which can be achieved by tuning the voltage, $\alpha$ and thereby $\omega / \Omega$. Then, one has

$$
T_{\gamma}(\delta=0)=\left|T_{\gamma}\right| e^{i \delta_{0} / 2}\left(\begin{array}{cc}
1 & 0 \\
0 & e^{-i \gamma}
\end{array}\right),
$$

and the unitary part of the transformation is a phase gate, ${ }^{3}$ where the phase difference introduced between spin up and spin down is just the geometrical angle $\gamma$ (Fig. 1). Figure 3 shows the curves along which such phase gates can be realized $[\delta=0$ in Eq. (13)] depending on the values of $k a$ and $\omega / \Omega$. The dots on the curves mark the points where $\left|T_{\gamma}\right|=1$, and thus the transformation is unitary. In Fig. 4 we show the gate efficiency of a phase gate $(\delta=0)$ for the special value $\gamma=\pi / 2$ as function of $\alpha$ and $k a$.

We note that in principle a number of other gates can be constructed by coupling several of those rings. This can be realized with parameters corresponding to unitary gates, so that the product of the corresponding spin rotations results again in a unitary transformation. For instance, two rings both with $\gamma=\pi / 2$ in Eq. (16) is a $Z$ gate. ${ }^{3}$ If such a gate is coupled to a diametric ring associated with Eq. (14) with $\theta=\pi / 4$, one obtains exactly a Hadamard gate. Similarly, two rings with $\gamma=\pi / 2$, plus two of the type corresponding to Eq. (14) with $\theta=\pi / 4$, yields a NOT gate with the correct phase.

As emphasized earlier, similar rings in the presence of an external magnetic field can be used for spin filtering. ${ }^{9}, 10$ This points to the possibility to integrate gates and filters that can serve as elementary building blocks of a quantum network based on spin sensitive devices. ${ }^{15-18}$
The present calculation was done for an idealized model system in which transport is ballistic and one dimensional, i.e., the finite width of the ring wire was not included. Currently, high mobility samples have become available such that at cryogenic temperatures transport is found to be ballistic over tens of microns. Similarly, phase coherence and spin coherence lengths ${ }^{19}$ have been found up to $100 \mu \mathrm{m}$. Our narrow ring implies the assumption of single-mode propagation. Recently, it was found that the finite width of the rings has a small effect on the loss of coherence of the spin state. ${ }^{10}$ Souma and Nikolić ${ }^{20}$ found that in a multichannel system the modulation of the transmitted spin states survives and that under specific conditions the individual eigenchannel transmissions are very similar to the ones found in single-channel rings. A possible nonideal coupling to the leads can be described through effective tunnel barriers. But, in most of the current experimental systems the leads are connected in a rather adiabatic way, which makes the coupling very close to ideal.

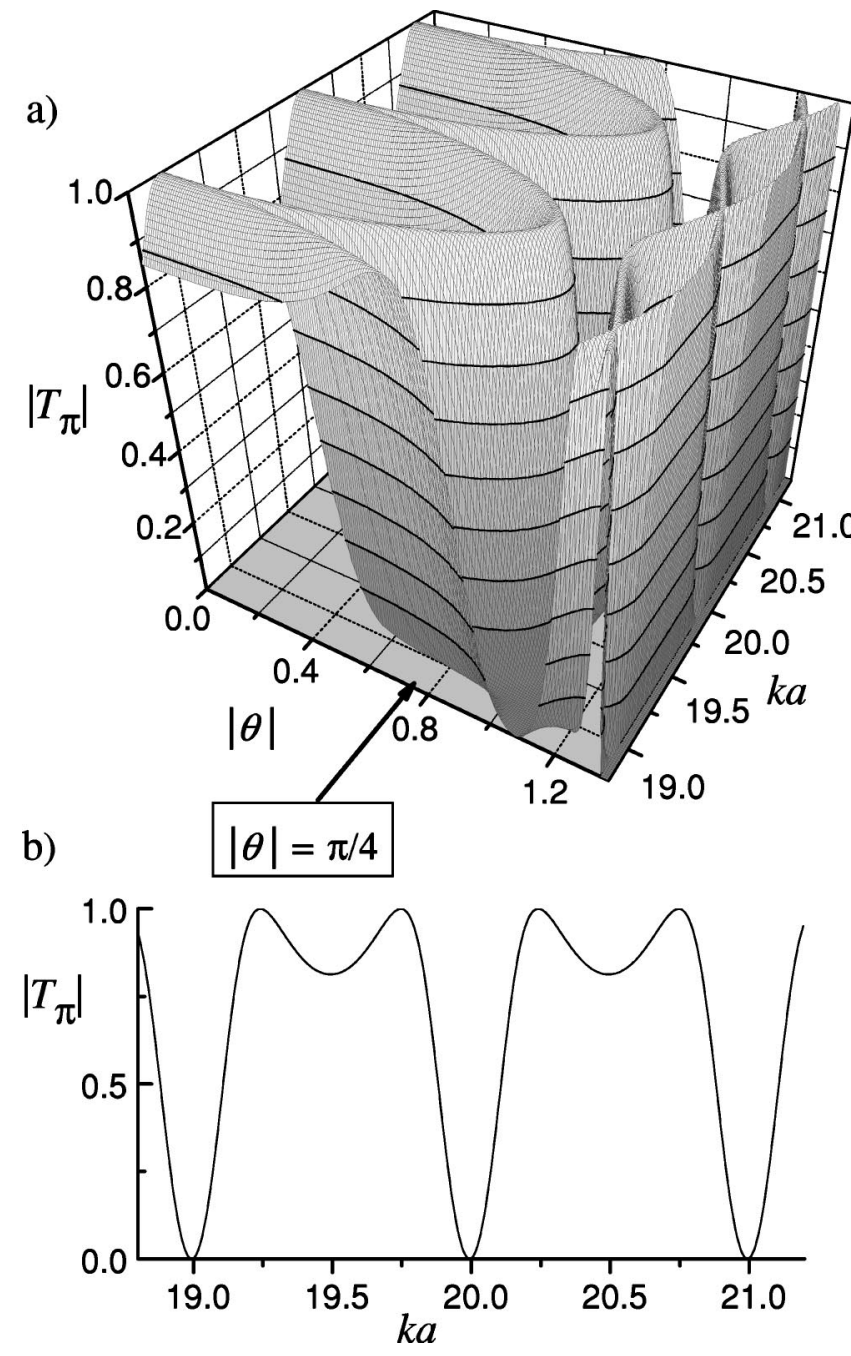

FIG. 2. (a) Efficiency $\left|T_{\pi}\right|$ of the quantum gate with $\gamma=\pi$ as a function of the modulus of the half rotation angle $\theta=-\arctan \omega / \Omega$ and $k a$. The maximal value of $|\theta|$ corresponds to $\omega / \Omega=3.5$. (b) Cross section of the surface at $|\theta|=\pi / 4$, where the transformation is essentially a Hadamard gate. 


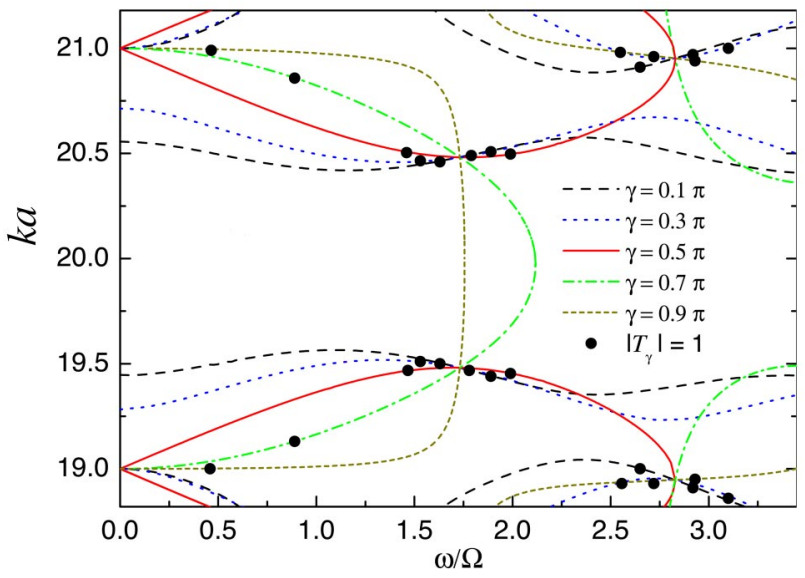

FIG. 3. (Color online) Lines along which the ring acts as a $\gamma$ phase gate. Points on the curves show where the gates are lossless, i.e., $\left|T_{\gamma}\right|=1$.

In conclusion, we have shown that a quantum ring with Rashba-type spin-orbit interaction can serve as a one-qubit quantum gate for electron spins. The spin transformation properties of the gates can be extended by coupling such rings in series. Different types of gates can be realized by tuning the electric field strength and changing the geometric position of the junctions connected to the ring, as well as by fabricating rings with different sizes. The considered parameters are within the experimentally feasible range. ${ }^{7,8,21}$ In a ring of radius $a=0.25 \mu \mathrm{m}$ and for $\operatorname{InGaAs}\left(m^{*}=0.023 \mathrm{~m}\right), \alpha$ can be varied ${ }^{7}$ up to $2.0 \times 10^{-11} \mathrm{eVm}$, which corresponds to $\theta=0.8(\pi / 2)$.

This work was supported by the Flemish-Hungarian Bilateral Programme, by the Flemish Science Foundation (FWO-Vl), by the Hungarian Scientific Research Fund

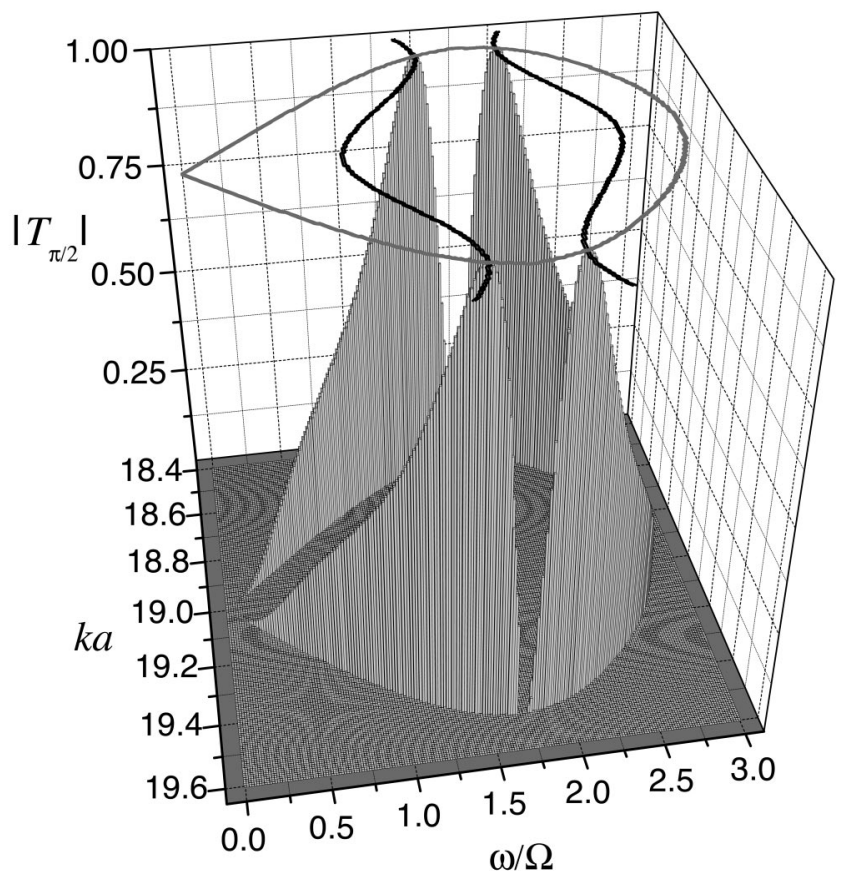

FIG. 4. Efficiency of a $\pi / 2$ phase gate. Along the gray curve on the top $(k a-\omega / \Omega)$ plane the ring acts as a $\pi / 2$ gate. The black lines on the same plane show where the efficiency $\left|T_{\pi / 2}\right|$ equals unity; thus, at the crossing points of the black and gray lines this phase gate is unitary.

(OTKA) under Contract Nos. D46043, M36803, and by the Hungarian Ministry of Education under Contract No. FKFP 099/2001. One of the authors (B.M.) is supported by the DWTC to promote $S \&$ T collaborations between Central and Eastern Europe.
${ }^{1}$ D. D. Awschalom, M. E. Flatté, and N. Samarth, Sci. Am. 286, 66 (2002).

${ }^{2}$ S. Datta and B. Das, Appl. Phys. Lett. 56, 665 (1990).

${ }^{3}$ M. A. Nielsen and I. L. Chuang, Quantum Computation and Quantum Information (Cambridge University Press, Cambridge, 2000).

${ }^{4}$ A. G. Aronov and Y. B. Lyanda-Geller, Phys. Rev. Lett. 70, 343 (1993).

${ }^{5}$ S. Viefers, P. Koskinen, P. S. Deo, and M. Manninen, Physica E (Amsterdam) 21, 1 (2004).

${ }^{6}$ E. I. Rashba, Sov. Phys. Solid State 2, 1109 (1960).

${ }^{7}$ J. Nitta, T. Akazaki, H. Takayanagi, and T. Enoki, Phys. Rev. Lett. 78, 1335 (1997).

${ }^{8}$ J. Nitta, F. E. Meijer, and H. Takayanagi, Appl. Phys. Lett. 75, 695 (1999)

${ }^{9}$ B. Molnár, F. M. Peeters, and P. Vasilopoulos, Phys. Rev. B 69, 155335 (2004).

${ }^{10}$ D. Frustaglia and K. Richter, Phys. Rev. B 69, 235310 (2004).
${ }^{11}$ F. E. Meijer, A. F. Morpurgo, and T. M. Klapwijk, Phys. Rev. B 66, 033107 (2002).

${ }^{12}$ S. Griffith, Trans. Faraday Soc. 49, 345 (1953).

${ }^{13}$ J. B. Xia, Phys. Rev. B 45, 3593 (1992).

${ }^{14}$ Y. Aharonov and A. Casher, Phys. Rev. Lett. 53, 319 (1984).

${ }^{15}$ J. C. Euges, G. Burkard, and D. Loss, Appl. Phys. Lett. 82, 2658 (2003).

${ }^{16}$ D. Stepanenko, N. E. Bonesteel, D. P. DiVincenzo, G. Burkard, and D. Loss, Appl. Phys. Lett. 68, 115306 (2003).

${ }^{17}$ J. B. Yau, E. P. DePoortere, and M. Shayegan, Phys. Rev. Lett. 88, 146801 (2003).

${ }^{18}$ D. Frustaglia, M. Hentschel, and K. Richter, Phys. Rev. Lett. 87, 256602 (2001).

${ }^{19}$ J. M. Kikkawa and D. D. Awschalom, Phys. Rev. Lett. 80, 4313 (1998).

${ }^{20}$ S. Souma and B. Nikolić, Phys. Rev. B 70, 195346 (2004).

${ }^{21}$ Y. Sato, S. G. T. Kita, and S. Yamada, J. Appl. Phys. 89, 8017 (2001). 